\title{
A GENERALIZATION OF A THEOREM OF P. MONTEL ON ENTIRE FUNCTIONS ${ }^{1}$
}

\section{CHUNG-CHUN YANG}

ABstract. This paper investigates the functional equation $a(z) f^{n}(z)+b(z) g^{m}(z)=1 \quad(a, b, f, g$ meromorphic).

In [4] P. Montel stated the following theorem:

THEOREM 1. Let $f(z), g(z)$ be two transcendental entire functions. Then if $m$ and $n$ are integers $\geqq 3$, the functional equation $f^{n}(z)+g^{n}(z)$ $=1$ cannot hold.

Recently, A. V. Jategaonkar [3] gave the complete proof, and obtained some analogous results for functions holomorphic in the unit disc. The purpose of this paper is to study the more general functional equations of the form $a(z) f^{n}(z)+b(z) g^{m}(z)=1$, where $n$, $m$ are integers greater than 3 .

It is assumed that the reader is familiar with the fundamental concepts of Nevanlinna's theory of meromorphic functions and the symbolism $m(r, f), N(r, f), T(r, f)$, etc.

THEOREM 1. The functional equation

$$
a(z) f^{n}(z)+b(z) g^{m}(z)=1
$$

$(a, b, f, g$ meromorphic, $m$ and $n$ integers $\geqq 3)$ cannot hold, if

$$
T(r, a(z))=\circ(T(r, f)), \quad T(r, b(z))=\circ(T(r, g)),
$$

unless $n=m=3$.

If $f(z)$ and $g(z)$ are entire and (2) holds, then (1) cannot hold, even if $n=m=3$.

Proof. We have for any meromorphic function $T\left(r, f^{n}\right) \sim n T(r, f)$. Therefore by (2),

$$
T\left(r, a f^{n}\right) \sim n T(r, f), \quad T\left(r, b g^{m}\right) \sim m T(r, g) .
$$

If (1) holds

Received by the editors October 31, 1969.

AMS 1968 subject classifications. Primary 3061.

Key words and phrases. Functional equations, Nevanlinna theory.

1 This work contains a generalization of the partial result which was contained in the author's doctoral thesis at the University of Wisconsin. 


$$
T\left(r, b g^{m}\right)=T\left(r, 1-a f^{n}\right) \sim T\left(r, a f^{n}\right) \sim n T(r, f) .
$$

Let

$$
\Theta(f, c)=1-\limsup _{r \rightarrow \infty} \frac{\bar{N}(r, c)}{T(r, f)},
$$

where $\bar{N}(r, c)$ is derived from the $c$-point of $f$ counted without regard to multiplicity.

Then $\bar{N}\left(a f^{n}, 0\right) \leqq \bar{N}(a, 0)+\bar{N}(f, 0)$, and by conditions (2) we have $\Theta\left(a f^{n}, 0\right) \geqq 1-1 / n$. Similarly $\Theta\left(a f^{n}, \infty\right) \geqq 1-1 / n$ and $\Theta\left(a f^{n}, 1\right)$ $=\Theta\left(b g^{m}, 0\right) \geqq 1-1 / m$.

According to Nevanlinna's second fundamental theorem (see, e.g. $[2$, p. 43]) we have

$$
\Theta\left(a f^{n}, 0\right)+\Theta\left(a f^{n}, 1\right)+\Theta\left(a f^{n}, \infty\right) \leqq 2 \text { or } 2 / n+1 / m \geqq 1 .
$$

Since $m, n \geqq 3$, the above inequality can hold only when $n=m=3$. The case $n=m=3$ can occur as shown, e.g. by F. Gross [1] when $a(z)=b(z)=1$. If $f$ is entire, $\Theta\left(a f^{n}, \infty\right)=1$ and we obtain $1 / n+1 / m \geqq 1$ which is impossible for $m, n \geqq 3$.

THEOREM 2. Let $f(z), g(z)$ and $f / g$ be transcendental meromorphic functions. Then

$$
h(z)=P(z) f^{n}(z)+q(z) g^{n}(z)
$$

$(p, q$ rational, $n$ integer $>3)$ has either infinitely many zeros or infinitely many poles.

Proof. Assume the statement is false. Then $h(z)=R(z) e^{n \alpha(z)}$, where $R(z)$ is a rational function, and $\alpha(z)$ is an entire function. Hence, we have

$$
\begin{aligned}
& R_{1}\left(f e^{-\alpha}\right)^{n}+R_{2}\left(g e^{-\alpha}\right)^{n}=1 ; \\
& T\left(r, R_{j}\right)=O(\log r) \quad(j=1,2) .
\end{aligned}
$$

At least one of $f e^{-\alpha}, g e^{-\alpha}$ has characteristic function $=O(\log r)$, i.e. at least one of $f e^{-\alpha}, g e^{-\alpha}$ is rational. But then the other is also rational, by (3). Contradiction to $\mathrm{f} / \mathrm{g}$ transcendental. The theorem follows from this.

REMARK. The proof of Theorem 1 can easily be adapted to obtain the analogous result for functions $f$ and $g$ which are meromorphic in the unit disc, as long as the analogous Nevanlinna's fundamental theorem for $f$ or $g$, and the growth conditions (2) are satisfied. 


\section{REFERENCES}

1. F. Gross, On the equation $f^{n}+g^{n}=1$, Bull. Amer. Math. Soc. 72 (1966), 86-88. MR 32 \#2595.

2. W. K. Hayman, Meromorphic functions, Oxford Mathematical Monographs, Clarendon Press, Oxford, 1964. MR 29 \#1337.

3. A. V. Jategaonkar, Elementary proof of a theorem of $P$. Montel on entire functions. J. London Math. Soc. 40 (1965), 166-170. MR 30 \#248.

4. P. Montel, Leçons sur les familles normales, Gauthier-Villars, Paris, 1927, pp. 135-136.

Michigan State University, East Lansing, Michigan 48823 